\title{
Impacto de la Pandemia por COVID-19 (SARS-CoV 2) en la Educación Odontológica: Scoping Review
}

\author{
Impact of the COVID-19 (SARS-CoV 2) Pandemic on Dental Education: Scoping Review
}

\author{
Joaquín Alexis Roco Bazáez
}

ROCO, B. J. A. Impacto de la pandemia por COVID-19 (SARS-CoV 2) en la educación odontológica: Scoping review. Int. J. Odontostomat., 15(1):10-13, 2021.

RESUMEN: El 11 de marzo de 2020 la OMS declara al COVID-19 como pandemia debido a los altos niveles de propagación a nivel mundial. Posteriormente la Administración de Seguridad y Salud Ocupacional (OSHA.gov) clasificó el quehacer odontológico como una actividad de alto riesgo por la potencial exposición y contagio por coronavirus SARS-CoV 2 debido a los procedimientos que generan aerosoles en virtud de la transmisión aérea del virus y contagio vía mucosa ocular, nasal u oral. Esto implicó el cese de las actividades odontológicas a nivel mundial. El siguiente artículo busca presentar el impacto de la pandemia por COVID-19 en la educación odontológica con base en la literatura electrónica disponible, además de analizar las diversas propuestas de aprendizaje a distancia asociado a la educación odontológica y los diversos medios tecnológicos para continuar con educación a distancia debido a la suspensión de las actividades teórica, preclínica y clínica.

PALABRAS CLAVE: Impacto, COVID-19, SARS-CoV 2, educación, odontología.

\section{INTRODUCCIÓN}

Consideraciones Generales. El 05 de enero de 2020 la Organización Mundial de la Salud (OMS) publica su primer parte sobre los brotes epidémicos relacionados a un nuevo virus. Desde aquí en adelante fueron aconteciendo una serie de sucesos debido a la expansión del virus, donde es así como el 12 de enero 2020 China hace pública la secuencia genética del virus causante de la enfermedad y el 30 de enero de 2020 la OMS por medio del Comité de Emergencias en virtud del reglamento sanitario internacional, declara el estado de "Emergencia de Salud Pública de Importancia Internacional" (ESPII), debido a que la infección afectaba a más de 18 países fuera de China. El 11 de febrero de 2020 la OMS anuncia que la enfermedad se denominará COVID-19 debido al nuevo coronavirus SARS-CoV 2 y ya el 19 de febrero 2020 se publican las consideraciones de cuarentena como medida de contención para evitar la transmisión del virus, para ya finalmente el 11 de marzo de 2020 declarar al COVID-19 como pandemia debido a los altos niveles de propagación a nivel mundial (Organización Mundial de la Salud, 2020a). Hoy a 19 de julio de 2020, el último reporte mundial de la OMS indica cerca de 14 millones de contagios por el virus SARS-CoV 2 y aproximadamente 600.000 mil muertes asociadas a éste (Organización Mundial de la Salud, 2020b).

De esta manera, la Administración de Seguridad y Salud Ocupacional (OSHA.gov) clasificó el quehacer odontológico como una actividad de alto riesgo debido a la potencial exposición y contagio por coronavirus SARSCoV 2 debido a los procedimientos que generan aerosoles. Esto implicó el cese de las actividades odontológicas a nivel mundial, teniendo un alto impacto a nivel formativo de la educación odontológica, tanto a nivel de pregrado como postgrado por la suspensión principal de actividades clínicas como teóricas, haciendo actualmente imprescindible la modificación de los métodos de enseñanza tradicionales considerando este tipo de situaciones, generando las condiciones necesarias para la continuación del aprendizaje constante teóricopráctico con base en protocolos de seguridad tanto para los pacientes como para el personal docente y alumnos.

El objetivo de este estudio fue revisar la literatura disponible sobre el impacto de la pandemia por COVID19 (SARS-CoV 2) en la educación odontológica y analizar las diversas propuestas de aprendizaje a distancia.

Facultad de Odontología sede Viña del Mar, Universidad Andrés Bello, Viña del Mar, Chile.

Recibido : 2020-07-20 Aceptado: 2020-09-16 


\section{MATERIAL Y MÉTODO}

Este estudio tuvo por diseño una revisión sistemática exploratoria o Scoping Review, con base en la guía de extensión de la declaración PRISMA (Preferred reporting ítems for systematic reviews and metaanalyses), PRIMS-ScR publicada en 2018, sobre el impacto de la pandemia por COVID-19 (SARS-CoV 2) en la educación odontológica, con base en los artículos electrónicos disponibles en PUBMED, Web of Science, Sciencedirect y Scielo. Para ello, se realizó búsqueda de la literatura utilizando los términos ("Impact"), ("dental education"), ("COVID-19") empleando el operador booleano AND. Como criterios de inclusión se aceptaron aquellos artículos tipo revisión sistemática (RS) con o sin metaanálisis (MA), publicados entre 01 de febrero 2020 y 01 de julio 2020, y que estuviesen en idioma inglés o español. Finalmente, como criterios de exclusión, aquellos artículos duplicados y manuscritos, cartas al editor y/o comentarios de especialistas.

La búsqueda fue realizada de manera independiente por el investigador J.A.R.B y esta última se hizo el 19 de julio de 2020.

\section{RESULTADOS}

Para el análisis de la síntesis cualitativa de este estudio se consideraron 5artículos que cumplieron con los criterios para ser incluidos.

Recolección de datos. La totalidad de artículos recolectados fueron identificados y filtrados sistemáticamente siguiendo el diagrama de flujo de la declaración PRISMA en conjunto con la aplicación de los criterios de inclusión y exclusión detallados anteriormente (Fig. 1).

\section{DISCUSIÓN}

Impacto de la pandemia por COVID-19 (SARS-CoV 2) en la educación odontológica. Los estudios reportan una disminución significativa a nivel mundial en la enseñanza y práctica odontológica asociada a la pandemia por COVID 19 (SARS-CoV 2), debido al alto riesgo que implica la generación de aerosoles para la transmisión aérea del virus y contagio vía mucosa ocu-

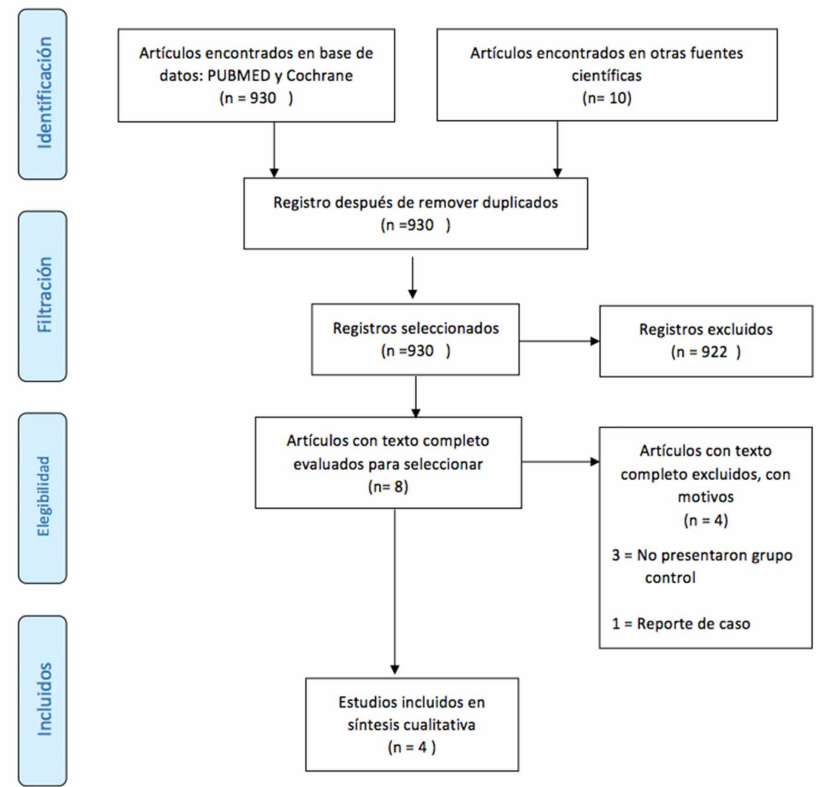

Fig. 1. Diagrama de flujo declaración PRISMA modificado. (Tricco et al., 2018).

lar, nasal u oral, además del contacto por superficies contaminadas, causando la cancelación de clases teóricas presenciales, actividades preclínicas en centros de simulación odontológica y actividades clínicas propiamente tal, dificultando la entrega del conocimiento teórico que sustenta las bases de los diagnósticos, indicación de exámenes complementarios y ejecución de tratamientos clínicos, afectando de manera importante la curva de aprendizaje del estudiante, posponiendo la atención de pacientes, clave en los planes de estudios de odontología general y sus especialidades (lyer et al., 2020).

Un estudio en CHINA, reporta que de 21 instituciones de educación continua dental antes de la pandemia por COVID-19 (SARS-CoV2) el $95.2 \%$ (20 de 21) impartía cursos de manera presencial u offline. Sin embargo, debido a la pandemia, todas las instituciones transfirieron sus cursos a una plataforma online, dando un aumento estadísticamente significativo (Liu, et al., 2020).

De igual manera, un estudio por la Asociación de Educación Dental en Europa, por medio de la evaluación de encuesta de cuestionario donde participaron 69 de 153 escuelas odontológicas de Europa, reportó que un $90 \%$ utiliza software online pedagógicos, donde un $72 \%$ realiza videos en streaming (Transmisión), un $48 \%$ por medio de link y/o material online y un $65 \%$ por medio de reuniones virtuales (Quinn et al., 2020). 
Metodología de enseñanza online. Es importante destacar el uso de plataformas digitales como ZOOM®, Microsoft TEAMS $®$, Jitsi®, WebEX®, etc, ya que permiten realizar las sesiones teóricas, demostraciones y evaluaciones a distancia. Esta modalidad puede ser sincrónica, por la cual el docente dicta una clase directamente a los alumnos permitiendo resolver las dudas en la sesión o asincrónica por la cual el docente graba la clase, encontrándose disponible para los alumnos, pero desarrollando las dudas de manera indirecta por medio de algún canal de comunicación. Si bien, la sesión presencial es la situación ideal, la modalidad online fomenta el autoaprendizaje, por otro lado, un punto crucial es la definición de protocolos de enseñanza online debido a que el éxito de la entrega de conocimiento teórico depende de la actitud e interacción entre el docente y los participantes (Barabari \& Moharamzadeh et al.).

De las actividades preclínicas y clínicas en situación de pandemia por COVID-19 (SARS-CoV 2). La formación preclínica y clínica se ha visto fuertemente afectada producto de la pandemia y las opciones digitales disponibles para realizarlas son escasas (Machado et al., 2020). A futuro es posible considerar el uso de realidad virtual (RV) en conjunto con la tecnología háptica basada en la aplicación del sentido táctil, ya que se sugiere que permitiría la adquisición de las habilidades psicomotoras como entrenamiento preclínico, además de entregarle una retroalimentación directa al docente (Barabari \& Moharamzadeh).

En algunas escuelas odontológicas de Europa se ha propuesto modificar los horarios y extender las horas clínicas de los programas (Machado et al.).

De las urgencias y emergencias odontológicas. La Asociación Dental Americana (ADA) recomendó la suspensión de toda actividad clínica entendiéndose como tratamientos electivos, dejando reservada la atención solo a urgencias y emergencias odontológicas (American Dental Association, 2020; lyer et al.).

En este punto, se podría considerar que el manejo de las urgencias odontológicas puede ser resuelta por un odontólogo general versus las emergencias que apuntan más hacia un manejo secundario por parte del especialista en cirugía y traumatología maxilofacial (Tabla I).

Situación en Chile. Para el desarrollo de esta revi-
Tabla I. Resumen general de Urgencias y emergencias odontológicas.

\begin{tabular}{cc}
\hline URGENCIAS & EMERGENCIAS \\
\hline Pericoronaritis & $\begin{array}{c}\text { Infección diseminada } \\
\text { tipo celulitis y/o } \\
\text { flegmón }\end{array}$ \\
sulintomática \\
Alveolitis & $\begin{array}{c}\text { Trauma maxilofacial } \\
\text { Hemorragia } \\
\text { incontrolable en } \\
\text { región orofacial }\end{array}$ \\
Abscesos & \\
odontogénicos & \\
Trauma & \\
dentoalveolar & \\
\hline
\end{tabular}

sión, actualmente no se encontraron estudios que hayan evaluado el impacto de la pandemia por covid19 (SARS-CoV2) en la educación odontológica a lo largo de Chile en las distintas universidades del país y/o institutos de formación técnica, sin embargo, teniendo en consideración el efecto producido en países desarrollados, podemos asumir que en Chile se produjo el mismo impacto. Además, este debe ser considerado no tan solo desde una vista netamente académica, sino que también desde la acreditación del pregrado y postgrado odontológico por la Comisión Nacional de Acreditación de Chile (2003, 2013), considerando el cumplimiento de los conocimientos mínimos necesarios definidos per se en el perfil de egreso y en los criterios de evaluación de esta entidad para la acreditación de las mismas.

Consideraciones a futuro. Las instituciones educacionales que imparten la odontología en nivel de pregrado y/o postgrado debiesen considerar lo siguiente en relación a futuras pandemias y/o eventos similares:

- Confección y actualización de protocolos en casos de contingencia como una futura pandemia, considerando el uso de tecnología para la mantención de las actividades teóricas y preclínicas.

- Confección y actualización de protocolos de infecciones asociadas a la atención en salud, con énfasis en el correcto uso de los equipos de protección personal (EPP).

- Mantención de la atención de urgencias y emergencias odontológicas, definiéndolas, categorizándolas y considerando la Teleodontología.

- Centrar el foco en la prevención y promoción de la salud oral. 


\section{CONCLUSIONES}

A nivel mundial se reportó un cese de las actividades teóricas, preclínicas y clínicas con excepción de algunas atenciones de urgencias y emergencias odontológicas, a causa de la expansión de la infección por COVID-19 (SARS-CoV 2) y la implementación de medidas de distanciamiento social y/o cuarentenas sanitarias.

Se debe definir, priorizar y reevaluar constantemente el desarrollo de protocolos de seguridad que resguarden la salud de los pacientes, personal docente y alumnos, así como también mantener la enseñanza en modalidad online, con el objetivo de asegurar la entrega permanente del conocimiento y mejorar las habilidades psicomotoras de los estudiantes, teniendo presente la aparición de futuras pandemias.

ROCO, B. J. A. Impact of the COVID-19 (SARS-CoV 2) pandemic on dental education: Scoping review. Int. J. Odontostomat., 15(1):10-13, 2021.

ABSTRACT: On March 11, 2020, the WHO declared COVID.19 as a pandemic due to high levels of spread worldwide. Later, the Occupational Safety and Health Administration (OSHA.gov) classified dental work as a highrisk activity due to the potential exposure and contagion by the SARS-CoV 2coronavirus due to the procedures that generate aerosols. This implied the cessation of dental activities worldwide. The following article seeks to present the impact of the COVID-19 pandemic on dental education based on the available electronic literature, in addition to analyzing the various distance learning proposals mainly associated with dental education and the various technogical means to continue with distance education due to the suspension of theoretical, preclinical and clinical activity.

KEY WORDS: Impact, COVID-19, SARS-CoV 2, education, odontology.

\section{REFERENCIAS BIBLIOGRÁFICAS}

American Dental Association. ADA develops guidance on dental emergency, nonemergency care. American Dental Association. Chicago, American Dental Association, 2020. Disponible en: https://www.ada.org/en/publications/ada-news/2020-archive/ march/ada-develops-guidance-on-dental-emergencynonemergency-care

Barabari, P. \& Moharamzadeh, K. Novel Coronavirus (COVID-19) and dentistry-A comprehensive review of literature. Dent. J. (Basel), 8(2):53, 2020.
Comisión Nacional de Acreditación (CNA). Criterios de Evaluación de Carreras de Odontología. Santiago de Chile, Comisión Nacional de Acreditación de Pregrado (CNAP), Comisión Nacional de Acreditación, 2003. Disponible en: https://www.cnachile.cl/ Criterios\%20de\%20carreras/odontologia.pdf

Comisión Nacional de Acreditación (CNA-CHILE). Criterios de Evaluación Para la Acreditación de Especialidades Odontológicas. Santiago de Chile, Comisión Nacional de Acreditación, 2013. Disponible en: https://www.cnachile.cl/Documentos $\% 20 \mathrm{de} \%$ 20Paginas/Criterios-de-Evaluaci\%C3\%B3n-para-laA creditaci \% C $3 \%$ B $3 n-d e-E s p e c i a l i d a d e s-$ Odontol\%C3\%B3gicas.pdf

Organización Mundial de la Salud (OMS). Cronología de la respuesta de la OMS a la COVID-19. Actualización 30 de junio 2020. Ginebra, Organización Mundial de la Salud, 2020a.

Organización Mundial de la Salud (OMS). Coronavirus disease (COVID-2019) Situation Reports 180. Ginebra, Organización Mundial de la Salud, 2020b. Disponible en: https://www.who.int/ docs/default-source/coronaviruse/situation-reports/20200718covid-19-sitrep-180.pdf?sfvrsn=39b31718_2

Iyer, P.; Aziz, K. \& Ojcius, D. M. Impact of COVID-19 on dental education in the United States. J. Dent. Educ., 84(6):718-22, 2020.

Liu, X.; Zhou, J.; Chen, L.; Yang, Y. \& Tan, J. Impact of COVID-19 epidemic on live online dental continuing education. Eur. J. Dent. Educ., 24(4):786-9, 2020.

Machado, R. A.; Bonan, P. R. F.; da Cruz Pérez, D. E. \& Martelli Júnior, H. COVID-19 pandemic and the impact on dental education: discussing current and future perspectives. Braz. Oral Res., 34:e083, 2020.

Quinn, B.; Field, J.; Gorter, R.; Akota, I.; Manzanares, M. C.; Paganelli, C.; Davies, J.; Dixon, J.; Gabor, G.; Mendes, R. A.; et al. COVID-19: the immediate response of european academic dental institutions and future implications for dental education. Eur. J. Dent. Educ., 2020. DOI: https://www.doi.org/10.1111/ eje. 12542

Tricco, A. C.; Lillie, E.; Zarin, W.; O'Brien, K. K.; Colquhoun, H.; Levac, D.; Moher, D.; Peters, M. D.; Horsley, T.; Weeks, L.; Hempel, S. et al. PRISMA extension for scoping reviews (PRISMA-ScR): checklist and explanation. Ann. Intern. Med., 169(7):467-73, 2018.

Dirección para correspondencia:

Joaquín Alexis Roco Bazáez

Licenciado en Odontología

Facultad de Odontología sede Viña del Mar

Universidad Andrés Bello

Viña del Mar

CHILE

Email: j.rocobazaez@gmail.com 